\title{
Internationale Gesellschaft für Gastro-Enterologie
}

Herr Professor Dr. Konjetzny, Direktor der Chirurgischen Universitäts-klinik und Poliklinik Hamburg-Eppendorf, bittet um Aufnahme folgender Notiz: Vom Präsidium der Internationalen Gesellschaft für Gastro-Entero-logie erhielt ich vor einiger Zeit die Mitteilung über die Beschlüsse des Komitees der Gesellschaft, das in Paris zusammengetreten war.

I. $\quad$ Der nächste Kongreß wird am 13., 14. und 15. September 1937

in Paris und am 16., 17. und 18. September 1937 in Vichy stattfinden.

II. Als Verhandlungsthemen sind gewählt

Frühdiagnose des Magenkarzinoms.

Akuter und chronischer Dünndarmverschluß.

Das erste Thema soil von Frankreich und Deutschland in Parallel-referaten behandelt werden. Als Vorsitzender des Deutschen National-Komitees bin ich beauftragt worden, die entsprechenden Referenten zu gewinnen.

Das zweite Thema ist den anderen Ländern zur Bearbeitung überantwortet worden.

V. Die Zahl der Mitglieder, welche für die Internationale Gesellschaft

für Gastro-Enterologie zugelassen wird, ist für Deutschland auf 150 fest-

gelegt worden. Der Jahresbeitrag beträgt 350 belgische Franken. Die

Meldung von Mitgliedern soil an mich gerichtet werden, da ich den Auftrag

habe, dann die deutsche Mitgliederliste insgesamt dem Kongreßbüro

einzusenden.

Es soil erreicht werden, daß die für Deutschland bestimmte Mitglieder-zahl voll ausgenutzt wird. Im Interesse der deutschen Belange liegt es, daß wir mit einer móglichst großen Mitgliederzahl in der Internationalen Gesellschaft für Gastro-Enterologie vertreten sind. 